AN. MED INTERNA (Madrid) Vol. 18, N. $^{\circ} 9$, pp. $455-458,2001$

\title{
El páncreas en la uremia
}

Herrero Calvo JA. El páncreas en la uremia. An Med Interna (Madrid) 2001; 18: 455-458.

En 1948 Baggenstoss describió por primera vez alteraciones histológicas pancreáticas en el $47 \%$ de las autopsias de 265 sujetos fallecidos por uremia crónica (1). Posteriormente otros estudios con autopsia de individuos fallecidos por insuficiencia renal crónica (IRC) y en hemodiálisis (HD) mostraron lesiones histológicas pancreáticas en el 56$60 \%$ de los casos (2-4). Los cambios histológicos consistían en ectasia ductal, fibrosis periductal, proliferación y metaplasia del epitelio ductular, acúmulo de material de secreción denso en las luces, atrofia acinar, engrosamiento y fibrosis arteriolar, depósitos de hemosiderina, depósitos cálcicos, cambios quísticos, amiloidosis, formación de microabcesos y necrosis de la grasa peripancreática. No está bien definido el origen de estos hallazgos histológicos, aunque se sospecha es multifactorial. Algunas de estas alteraciones pueden ser explicadas en el contexto general de complicaciones del paciente urémico, tales como la amiloidosis por depósito de $\beta_{2}$-microglobulina, o los depósitos de hemosiderina en pacientes politransfundidos en la era previa al empleo de eritropoyetina (5), sin embargo, la mayoría de las lesiones que son compatibles con pancreatitis crónica, no tienen una patogenia conocida. La hipercalcemia es una causa conocida de pancreatitis. Por otra parte se sabe que el hiperparatiroidismo secundario es una de las causas más frecuentes de calcificaciones en los enfermos con insuficiencia renal crónica (6). Se ha sugerido que el hiperparatiroidismo secundario puede ser el responsable de las alteraciones histológicas pancreáticas en el paciente con IRC (7) aunque no se ha podido demostrar una relación entre calcificaciones pancreáticas y lesiones de pancreatitis crónica (3). Owyan y cols. refirieron un aumento de colecistocinina (CCK) y otras hormonas gastrointestinales en los pacientes con IRC, aumento que guardaba relación con el grado de insuficiencia renal (8). En otro trabajo posterior describieron en pacientes urémicos una hipersecreción de tripsina en el aspirado duodenal en respuesta a la estimulación con CCK, así como un aumento en la vida media plasmática de la CCK administrada (9). Los autores especulan que la estimulación crónica del páncreas exocrino por la CCK podría ser responsable de estas lesiones. Esta hipótesis sin embargo, no está avalada por las observaciones de otros autores que contrariamente describen en los pacientes en HD, una hipofunción más que una hiperfunción del páncreas exocrino. Así, Ventrucci y cols. encuentran una disminución en la excreción fecal de quimiotripsina y de elastasa 1 en el $30 \%$ de los pacientes en HD $(10,11)$. También se ha descrito en pacientes dializados una disminución de la secreción pancreática de amilasa y lipasa con la prueba de secretina-CCK (12). En cualquier caso la repercusión clínica de las lesiones pancreáticas crónicas es escasa o al menos difícilmente detectable. Los pacientes con disminución del contenido de quimiotripsina y elastasa 1 descritos por Ventrucci y cols. estaban asintomáticos y la ecografía del páncreas era normal $(10,11)$. En el estudio con autopsia de Vaziri y cols no se encontró relación entre las anormalidades histológicas y evidencia de enfermedad pancreática durante la estancia en diálisis, como por ejemplo pancreatitis aguda o crónica, o insuficiencia del páncreas exocrino (3).

En 1985 fueron publicados dos casos de pancreatitis aguda en pacientes que estaban en tratamiento con diálisis peritoneal contínua ambulatoria (DPCA) (13). Poco después fueron referidos 4 nuevos casos de pacientes también en programa de DPCA (14). Estudios posteriores han confirmado que los enfermos crónicos tratados con DPCA y $\mathrm{HD}$, tienen un riesgo superior de desarrollar pancreatitis aguda respecto a la población general (15-19). Aunque con excepciones (16), la mayoría de las observaciones apuntan que la incidencia de pancreatitis aguda es significativamente superior en los pacientes en DPCA que en los de HD $(15,18,19)$. De esta manera, la incidencia por 100 pacientes año oscila en el rango de 0.16-1.41 en HD y de 0,46-4,3 en DPCA (15,17-19). La evolución clínica y por tanto el pronóstico de la pancreatitis aguda en estos paciente es llamativamente distinta en las series publicadas. Así, se ha referido 
una mortalidad del 4\% (18), $11 \%$ (19), $20 \%$ (15), 33\% (20) y hasta del $58 \%(17)$, que puede ser similar o muy superior a la población general $(21,22)$.

Los mecanismos patogénicos responsables del mayor riesgo de pancreatitis aguda en los pacientes en diálisis no han sido claramente identificados. Teóricamente los enfermos dializados crónicamente tienen una serie de factores de riesgo adicionales a la población general, como es la propia uremia, el hiperparatiroidismo secundario, la hipertrigliceridemia o fármacos, sin embargo, en los estudios realizados no se han encontrado diferencias en ninguno de estos factores cuando se comparaban los pacientes que presentaban pancreatitis aguda con el resto de la población en diálisis (15,18-20). El tiempo de permanencia en diálisis (HD y DPCA) y el antecedente de peritonitis aguda en pacientes en DPCA, no parecen ser factores que condicionen mayor riesgo $(18,19)$. En los pacientes en DPCA se ha especulado que la infusión de una gran cantidad de líquido con una composición no fisiológica, que causa un incremento no fisiológico de la presión intra-abdominal, podría hacer al páncreas más susceptible de daño parenquimatoso (19). El aumento de presión podría lesionar la microvascularización y causar hipooxia, la cual podría inducir una activación de los enzimas proteolíticos y provocar pancreatitis. Por otro lado, la alta concentración de glucosa en el líquido de diálisis peritoneal predispone a la hipertrigliceridemia lo que podría ser un riesgo añadido. En este momento se desconoce si otras técnicas de diálisis peritoneal distintas de la DPCA, como la diálisis peritoneal intermitente nocturna con máquina cicladora automática, en la que la presión intra-abdominal es menor ya que el paciente está en decúbito y durante el día la cavidad peritoneal está vacía, o el empleo de los nuevos líquidos de perfusión libres de glucosa, modifican de alguna manera la frecuencia de aparición de pancreatitis aguda en estos pacientes.

Un aspecto importante a considerar en el diagnóstico de pancreatitis aguda en los enfermos urémicos, es que en ausencia de pancreatitis, los niveles séricos de enzimas pancreáticos están frecuentemente elevados en los pacientes con IRC no dializados y en pacientes en diálisis, tanto HD como diálisis peritoneal. Aunque no puede descartarse que la elevación enzimática sea consecuencia de daño pancreático crónico, la opinión general es que está producida por un defecto en la eliminación renal, dado que por un lado las enzimas están tambíen aumentadas en el fracaso renal agudo $(23,35)$, y por otro, que su aumento se relaciona con el grado de insuficiencia renal en los pacientes crónicos (36-39). Collen y cols. refieren elevaciones de amilasa, lipasa y tripsina únicamente en aquellos pacientes con aclaramiento de creatinina inferior a $50 \mathrm{ml} / \mathrm{min}$ (38). En el grupo estudiado por Seno y cols., había elevación enzimática significativamente diferente de los controles en los pacientes con aclaramiento de creatinina inferior a $40 \mathrm{ml} / \mathrm{min}$ y a medida que la insuficiencia renal era mayor, la frecuencia de aumento de las enzimas también se incrementaba (39). Con aclaramiento de creatinina inferior a $10 \mathrm{ml} / \mathrm{min}$ el $85,7 \%$ de los pacientes tenían valores aumentados de la amilasa, el 71,4\% de la lipasa, el $100 \%$ de la tripsina, el $100 \%$ de la fosfolipasa $\mathrm{A}_{2} \mathrm{y}$ el $42,9 \%$ de la elastasa 1 . Estas elevaciones enzimáticas suelen ser moderadas, en torno a 2,5 veces el valor normal, aunque se han descrito valores de amilasa muy superiores, hasta 7 veces por encima de los valores de referencia (40).
No está suficientemente aclarado el mecanismo fisiopatogénico por el cual se produce una disminución de la eliminación renal de enzimas pancreáticos en la IRC, ni tampoco de la diferente vulnerabilidad de las distintas enzimas al daño renal. Se ha sugerido que la disminución o pérdida de la carga negativa glomerular podría jugar un papel importante en estos fenómenos. En sujetos normales la tripsina en sangre está en su mayoría como tripsinógeno libre con una proporción de 2:1 de la forma catiónica respecto a la aniónica $(41,42)$. La forma catiónica, que es la que se mide en las determinaciones analíticas, sería la más susceptible ante una pérdida de la carga negativa glomerular $(43,44)$. La fosfolipasa $\mathrm{A}_{2}$ se encuentra en sangre también en forma libre y en condiciones de normalidad es fácilmente filtrada por el glomérulo $(45,46)$. Existen datos contradictorios a cerca de la distinta vulnerabilidad de la amilasa y lipasa al daño renal. Algunos autores refieren que la lipasa se eleva en una menor proporción de pacientes que la amilasa $(24,39)$, mientras que otros no encuentran diferencias $(29,30)$ o incluso mayor porcentaje de enfermos con aumento de la lipasa (47). Los niveles plasmáticos de elastasa 1 son los que menos se afectan por la insuficiencia renal ya que se elevan sólo si el aclaramiento de creatinina es inferior a 10 $\mathrm{ml} / \mathrm{min}$ (39). La elastasa 1 está presente en la sangre como formas complejas con inhibidores proteicos como la $\alpha_{1^{-}}$ antitripsina y la $\alpha_{2}$-macroglobulina (48) las cuales se filtran mal por el glomérulo y son aclaradas por vías metabólicas extrarrenales (49). Se desconoce si en la uremia se produce algún daño de esas vías metabólicas, lo que de producirse justificaría su elevación.

La mayoría de los autores refieren aumentos similares de los niveles séricos de amilasa y lipasa en los pacientes en HD y en DPCA $(24,38,50,51)$, a pesar de que la producción extra-pancreática de lipasa puede incrementarse en los pacientes en HD por el efecto de la heparina administrada durante la sesión $(52,53)$. En otro trabajo se describe que tanto los niveles de lipasa, como los de amilasa total y amilasa pancreática eran significativamente mayores en HD que en DPCA (34). La capacidad depurativa no es factor determinante ya que tanto en HD como en DPCA los aclaramientos de los diversos enzimas pancreáticos por el líquido de diálisis son insignificantes $(34,54,55)$. Las elevaciones enzimáticas persisten estables en el tiempo, como se ha referido tras 4 años de seguimiento en un grupo de pacientes estables en HD (10).

En general se asume que el diagnóstico de pancreatitis aguda en los pacientes urémicos es de alta sospecha, cuando unido a la sintomatología los niveles séricos de los enzimas pancreáticos exceden 3 veces el valor normal $(38,39)$. Sin embargo, en ausencia de pancreatitis han sido referidos aumentos mayores en numerosas observaciones $(34,40,51)$. La determinación de proteinas específicas pancreáticas, como la procarboxipeptidasa B no es de utilidad, dado que también está aumentada en los pacientes con insuficiencia renal (56). La determinación de isoenzimas es de gran ayuda diagnóstica. En los pacientes con IRC con o sin diálisis se produce elevación tanto de la amilasa pancreática como de la amilasa de producción extrapancreática principalmente salival, dado que comparten la misma vía de eliminación renal $(25,36,38,51)$. En pacientes con síntomas sugestivos de pancreatitis aguda la desproporción en la elevación de la amilasa pancreática respecto a la salival orienta al diagnós- 
tico. En un interesante trabajo publicado en este número se demuestra la utilidad de la determinación de las distintas isoamilasas pancreáticas y de las isolipasas en los pacientes con fracaso renal agudo e IRC en HD (57). Cabe señalar también que recientemente se ha descrito que la icodextrina, un polímero de la glucosa derivado del almidón, empleado en la composición de los líquidos de diálisis peritoneal como sustituto de la glucosa, interfiere en el método enzi- mático de determinación de amilasa total aunque no de la lipasa (58).

\section{J. A. HERRERO CALVO \\ Servicio de Nefrología. Hospital Clínico San Carlos. Madrid.}

\section{Bibliografía}

1. Baggenstoss $\mathrm{AH}$. The pancreas in uremia: a histopathologic study. Am J Pathol 1948; 24: 1003-11.

2. Avram MM. High prevalence of pancreatic disease in chronic renal failure. Nephron 1977; 18: 68-72.

3. Vaziri ND, Dure-Smith B, Miller R, Mirahmadi MK. Pancreatic pathology in chronic dialysis patients -an autopsy study of 78 cases. Nephron 1987; 46: 347-9.

4. Araki T, Ueda M, Ogawa K, Tsuji T. Histological pancreatitis in endstage renal disease. In J Pancreatol 1992; 12: 263-9.

5. Elias D, Varizi ND, Farooqui S, Mirahmadi MK, Martin DC. Pathology of endocrine organs in chronic renal failure. In J Artif Organs 1984; 7 : 251-6.

6. Llach F, Bover J. Renal osteodystrophies. In: Brenner BM, editor. Brenner and Rector's The Kidney. 6nd ed. Philadelphia: W.B. Saunders Company; 2000; 465-78.

7. Avram MM, Iancu M. Pancreatic disease in uremia and parathyroid hormone excess. Nephron 1982; 32: 60-4.

8. Owyang C, Miller LJ, Brennan LA, Go VLW. Gastrointestinal hormone profile in renal insufficiency. Mayo Clin Proc 1979; 54: 769-73.

9. Owyang C, Miller LJ, DiMagno EP, Mitchell JC, Go VLW. Pancreatic exocrine function in severe human chronic renal failure. Gut 1982; 23: 357-61.

10. Ventrucci M, Campieri C, Di Stefano M, Ubalducci GM, Li Bassi S, Di Grazia A, et al. Alterations of exocrine pancreas in end-stage renal disease. Do they reflect a clinically relevant uremic pancreopathy? Dig Dis Sci 1995; 40: 2576-81.

11. Ventrucci M, Cipolla A, Middonno M, Racchini C, Simoni P, Afandi K, et al. Impaired fecal elastase excretion in uremic pancreopathy. Dig Dis Sci 2000; 45: 2265-9.

12. Sachs EF, Hurwitz FJ, Bloch HM, Milne FJ. Pancreatic exocrine hypofuntion in the wasting syndrome of end-stage renal disease. Am J Gastroenterol 1983; 78: 170-3

13. Pitrone F, Pellegrino E, Mileto G, Consolo F. May pancreatitis represent a CAPD complication? Report of two cases with a rapidly evolution to death. In J Artif Organs 1985; 8: 325.

14. Caruana RJ, Wolfman NT, Karstaedt N, Wilson DJ. Pancreatitis: an important cause of abdominal symptoms in patients on peritoneal dialysis. Am J Kidney Dis 1986; 7: 135-40.

15. Rutsky EA, Robards M, Van Dyke JA, Rostand SG. Acute pancreatitis in patients with end-stage renal disease without transplantation. Arch Intern Med 1986;146: 1741-5.

16. Gupta A, Yuan ZY, Balaskas EV, Khannar R, Oreopoulos DG. CAPD and pancreatitis: no connection. Perit Dial Int 1992; 12: 309-16.

17. Pannekeet MM, Kredier RT, Boeschoten EW, Arisz L. Acute pancreatitis during CAPD in The Netherlands. Nephrol Dial Transplant 1993; 8: 1376-81.

18. Padilla B, Pollak VE, Pesce A, Kant S, Gilinsky NH, Deddens JA. Pancreatitis in patients with end-stage renal disease. Medicine (Baltimore) 1994; 73: 8-20.

19. Bruno MJ, van Westerloo DJ, van Dorp WT, Dekker W, Ferwerda J, Tytgat GNJ, et al. Acute pancreatitis in peritoneal dialysis and haemodialysis: risk, clinical course, outcome, and possible aetiology. Gut 2000; 46: 385-9.

20. Pitchumoni CS, Arguello P, Agarwal N, Yoo J. Acute pancreatitis in chronic renal failure. Am J Gastroenterol 1996; 91: 2477-81.
21. Halvorsen FA, Ritland S. Acute pancreatitis in Buskerud Country, Norway. Incidence and etiology. Scand J Gastroenterol 1996; 31: 411-4.

22. Eland IA, Sturkenboon MJCM, Wilson JHP, Stricker BH Incidence and mortality of acute pancreatitis between 1985 and 1995. Scand J Gastroenterol 2000; 35: 1110-6

23. Hörl WH, Wanner C, Schollmeyer P, Ogawa M. Plasma levels of pancreatic secretory trypsin innhibitor in relation to amylase and lipase in patients with acute and chronic renal failure. Nephron 1988; 49: 33-8.

24. Royse VL, Jensen DM, Corwin HL. Pancreatic enzymes in chronic renal failure. Arch Intern Med 1987; 147: 537-9.

25. Berk JE, Fridhandler L, Ness RL. Amylase and isoamylase activities in renal insufficiency. Ann Intern Med 1979; 90: 351-3.

26. Bailey GL, Katz AL, Hampers CL, Merril JP. Alterations in serum enzymes in chronic renal failure. JAMA 1970; 213: 2263-5.

27. Pedersen EB, Brock A, Kornerup HG. Serum amylase activity and renal amylase activity clearance in patients with severely impaired renal function and in patients treated with renal allotransplantation. Scand J Clin Lab Invest 1976; 36: 137-40.

28. Fahrenkrug J, Staun-Olse P, Magid E. Immunoreactive trypsin and pancreatic isoamylase activity in serum of patients with chronic renal failure or hepatic cirrhosis. Clin Chem 1981; 27: 1655-7.

29. Montalto G, Carroccio A, Sparacino V, Lorella D, Di Martino D, Soresi $\mathrm{M}$, et al. Pancreatic enzymes in chronic renal failure and transplant patients. Int J Pancreatol 1992; 12: 211-7.

30. Montalto G, Lorello D, Carroccio A, Sparacino V, Li Vecchi M, Soresi $\mathrm{M}$, et al. Serum trypsin in chronic renal failure and transplant patients. Am J Gastroenterol 1992; 87: 1175-9.

31. Vaziri ND, Chang D, Malekpour A, Radaht S. Pancreatic enzymes in patients with end-stage renal disease maintained on hemodialysis. Am J Gastroenterol 1988; 83: 410-2.

32. Araki T, Ueda M, Taketa K, Kosaka K. Pancreatic-type hyperamylasemia in end-stage renal disease. Dig Dis Sci 1989; 34: 1425-7.

33. Manes G, Ricci E, Rabitti PG, Caruso S, Carraturo I, Laccetti, et al Serum pancreatic enzymes in patients with chronic renal failure on hemodialysis and in transplant patients. Minerva Gastroenterol Dietol 1994; 40: 113-7.

34. Masoero G, Bruno M, Gallo L, Colaferro S, Cosseddu D, Vacha GM. Increased serum pancreatic enzymes in uremia: Relation with treatment modality and pancreatic involment. Pancreas 1996; 13: 350-5.

35. Zachee P, Lins RL, De Broe ME. Serum amylase and lipase values in acute renal failure. Am J Gastroenterol 1985; 31: 1237.

36. Morton WJ, Tedesco FJ, Harter HR, Alpers DH: serum amylase determinations and amylase to creatinine clearance ratios in patients with chronic renal insufficiency. Gastroenteroly 1976; 71: 594-8.

37. Buchman A, Ament ME, Moukarzel A. Total serum amylase but not lipase correlates with measured glomerular filtration rate. J Clin Gastroenterol 1993; 16: 204-6.

38. Collen MJ, Ansher AF, Chapman AB, Mackow RC, Lewis JH. Serum amylase in patients with renal insufficiency and renal failure. Am J Gastroenterol 1990; 85: 1377-80.

39. Seno T, Harada H, Ochi K, Tanaka J, Matsumoto S, Choudhury R, et al. Serum levels of six pancreatic enzymes as related to the degree of renal dysfunction. Am J Gastroenterol 1995; 90: 2002-5.

40. Levitt MD, Ellis C. Serum isoamylase measurements in pancreatitis complicating chronic renal failure. J Lab Clin Med 1979; 93: 71-7. 
41. Kimland M, Russick C, Marks WH, Borgstrom A. Immunoreactive anionic and cationic trypsin in human serum. Clin Chim Acta 1989; 184: 31-46.

42. Abu-Alfa A, Ivanovich P, Mujais SK. Uremic exocrine pancreopathy. Nephron 1988; 48: 94-100.

43. Fabris C, Benini L, Del Favero G, Cavallini G, Basso D, Vantini I, et al. Molecular size distribution of immunoreactive trypsin and renal tubular dysfunction: Role in trypsin plasma-urine transfer. Enzyme 1987; 37: 174-81.

44. Fabris C, Benini L, Basso D, Del Favero G, Vantini I, Piccoli A, et al. Renal factors in serum trysinogen I metabolism and excretion in chronic pancreatic disease. Pancreas 1988; 3: 25-9.

45. Fabris C, Basso D, Panozzo MP, Del Favero G, Meggiato T, Plebani M, et al. Urinary phospholipase A2 excretion in chronic pancreatic diseases. Int J Pancreatol 1992; 11: 179-84.

46. Funakoshi A, Yamada Y, Ito T, Ishikawa H, Yokota M, Shinozaki H, et al. Clinical usefulness of serum phospholipase A2 determination in patients with pancreatic diseases. Pancreas 1991; 6: 588-94.

47. Malfertheiner P, Büchler M, Stanescu A, Uhl W, Ditschuneit H. Serum elastase 1 in inflammatory pancreatic and gastrointestinal diseases and in renal insufficiency: a comparison with other serum pancreatic enzymes. Int J Pancreatol 1987; 2: 159-70.

48. Murata A, Ogawa M, Fujimoto K, Kitahara T, Matsuda Y, Kosaki G. Radioimmunonoassy of human pancreatic elastase 1. Enzyme 1983; 30: 29-37.

49. Fabris C, Basso D, Benini L, Meggiato T, Del Favero G, Cavallini G, et al. Urinary elastase 1 in chronic pancreatic disease. Enzyme 1989; 42: 80-6.
50. Lin XZ, Chen TW, Wang SS, Shiesh SC, Tsai YT, Huang TP, et al. Pancreatic enzymes in uremic patients with or without dialysis. Clin Biochem 1988; 21: 189-92.

51. Tsianos EV, Dardamanis MA, Elisaf M, Vasakos S, Siamopoulos KC. The value of alpha-amylase and isoamylase determination in chronic renal failure patients. In J Pancreatol 1994; 15: 105-11.

52. Crawford GA, Savdie E, Stewart JH. Heparin-released plasma lipase in chronic renal failure and after renal transplantation. Clin Sci 1979; 57: 155-65.

53. Montalto G, Soresi M, Carroccio A, Galione A, Lorello D, Di Martino $\mathrm{D}$, et al. Influence of haemodialysis on lipase activity. Eur J Clin Chem Biochem 1997; 35: 237-8.

54. Caruana RJ, Burkart J, Segraves D, Smallwood S, Haymore J, Disher B. Serum and peritoneal fluid amylase levels in CAPD. Normal values and clinical usefulness. Am J Nephrol 1987; 7: 169-72.

55. Bastani B, Mifflin TE, Lowell MA, Westervelt FB, Bruns DE. Serum amylase in chronic and end-stage renal failure: effects of mode of therapy, race, diabetes and peritonitis. Am J Nephrol 1987; 7: 292-9.

56. Chen CC, Wang SS, Chen TW, Jap TS, Chen SJ, Jeng FS, et al. Serum procarboxypeptidase $\mathrm{B}$, amylase and lipase in chronic renal failure. $\mathrm{J}$ Gastroenterol Hepatol 1996; 11: 496-9.

57. Sánchez Navarro MR, Fernández-Conde E, Samaniego C. Valoración de isoamilasas e isolipasas en el suero de pacientes con insuficiencia renal. An Med Interna (Madrid) 2001; 18: 459-63.

58. Schoenicke G, Grabensee B, Plum J. Interference of icodextrin with serum amylase activity. Perit Dial Int 2001; 21 (suppl 1): S31 (abstract). 